\title{
Shear Rate and Permeability in Water Flooding
}

\author{
Nico Reuvers • Michael Golombok
}

Received: 5 June 2008 / Accepted: 25 November 2008 / Published online: 23 December 2008

(C) The Author(s) 2008. This article is published with open access at Springerlink.com

\begin{abstract}
Water breakthrough during oil recovery can be inhibited by the use of novel shear-induced structure additives. These affect the shear rate experienced by fluid inside the rock matrix. We relate this to the permeability for the purpose of applying these novel non-Newtonian materials which thicken viscosity only in the shear range associated with the permeability of the reservoir while maintaining base fluid properties outside this range.
\end{abstract}

Keywords Water flooding $\cdot$ Permeability $\cdot$ Shear rate

\section{Symbols}

$\mathrm{k}$ Pre-multiplying constant in power law non-Newtonian response

$n \quad$ Index in power law non-Newtonian response

$p$ Pressure

$x \quad$ Axial length

$u$ Darcy velocity

$\gamma$ Shear rate

$\varphi$ Porosity

$\kappa \quad$ Permeability

$\mu$ Dynamic viscosity

Liquids which display non-Newtonian flow have long been proposed as a solution for the non-uniform injected water flow in an oil reservoir during secondary recovery (Jones 1980). In particular, shear thickening represents a method for retarding the flow in more permeable

N. Reuvers · M. Golombok $(\bowtie)$

Department of Mechanical Engineering, Technische Universiteit Eindhoven, 5600 MB, Eindhoven, The Netherlands

e-mail: m.golombok@tue.nl

M. Golombok

Shell International Exploration and Production,

Kessler Park 1, 2288 GS, Rijswijk, The Netherlands 
Fig. 1 Flow profiles for water passing from the injector well (I) to the producer well $(\mathrm{P})$ in a reservoir schematicised here as consisting of a highly permeable region $\left(\kappa_{\mathrm{hi}}\right)$ sandwiched between two low permeability regions $\left(\kappa_{\mathrm{lo}}\right): 1-$ The ideal flat front profiles; 2 -The deviating breakthrough profile; 3 - The retarded profile under the influence of shear induced structure additives

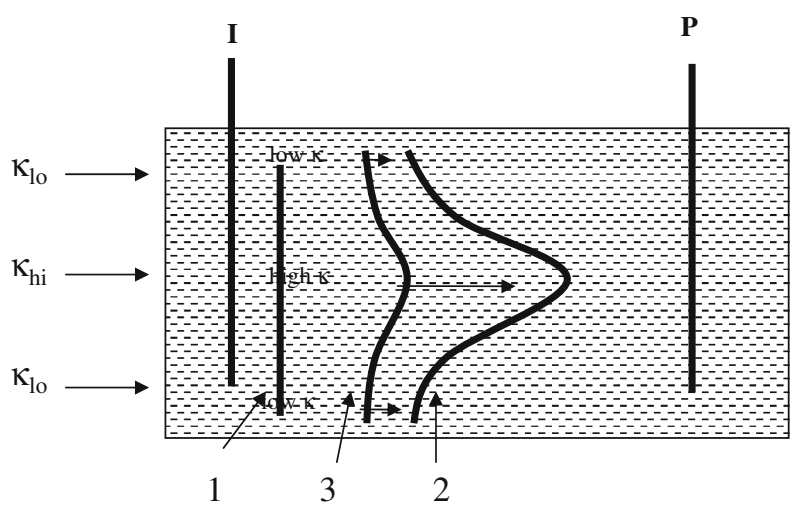

regions of an oil reservoir during water flooding for oil extraction. The problem is shown schematically in Fig. 1. Here the oil reservoir problem is simplified for illustrative purposes and is presented schematically as a central high permeability layer, sandwiched between two low permeability regions. Ideally, we wish that an injected water front proceeds uniformly through the reservoir (indicated by profile 1). However, due to permeability variations, the water front advances in the high permeability regions (profile 2). Eventually, the water front breaks through into the producer bore, and the low permeability regions are not swept. This breakthrough point can be retarded if the viscosity can be made to increase in the high permeability region. (In this regard, we ignore for the time being the relative permeability since we are concerned with controlling the water flow over the spatially varying permeability structure.) We have recently experimentally demonstrated how an additive can induce profile flattening (profile 3) (Golombok et al. 2008). In this note we examine the interaction between fluid shear and rock permeability, which seems to govern this effect.

Shear thickening is governed by the applied shear rate. Our analysis in this study is not based on a detailed description of the microscopic or cellular physics of flow in porous media, but rather on the physical empirical parameters associated with the phenomena. In this case, the two variables are:

1) the shear rate $\gamma$, in classic fluid flow experiments this can be imposed, for example, as in a classic Couette cell where rotation rates across a fixed gap determine the value of the shear rate. The definition in porous media is less clear and is partially the subject of this article.

2) the permeability $\kappa$ : the classic Darcy law definition of flow through a porous medium under an applied pressure drop.

Shear thickeners have not found any application to date in improved oil recovery for two reasons. First, viscosity $\mu$ typically increases monotonically as a function of shear rate $\gamma$ such as exhibited in classical power law behaviour

$$
\mu=k \gamma^{n-1}
$$

Even if the viscosity modifying agent is injected downhole, and although theoretically favourable behaviour may be exhibited in the reservoir, the flow is stopped in the producer well bore where the shear rate is the highest. Second, the concentrations of traditional shear thickeners required are typically $10-25 \% \mathrm{v} / \mathrm{v}$ making it too expensive.

The essential physical principle behind using shear thickeners remains valid and is conceptually very simple. Shear rate is the imposed velocity gradient perpendicular to axial flow, 
whereas permeability is (to first order) a locally varying property of the rock matrix through which flow occurs (Turcott and Schubert 1982). The relationship between the two can be seen by relating a high permeability to a large diameter capillary pipe and a low permeability to a small diameter pipe. A comparison with Darcy's law shows that any observed permeability is related to the pressure gradient within the pores which are modeled as capillaries. However, rather than pursuing this route, we ground our study in a number of recently reported experimental results relating to pressure drop and the resulting fluid velocity when unusual shear induced structure materials are added.

Figure 2 summarises these results (Golombok et al. 2008). The straight line shows the classic linear Darcy response in a porous rock sample where the observed velocity is linear with the applied pressure drop. When small millimolar concentrations of shear-induced structure chemicals (such as cetyl trimethyl ammonium bromide and sodium salicylate) are added to the water, then the positively deviating curve in Fig. 2 is observed. At any reference pressure, the flow is retarded, and this happens selectively in high permeability rocks. The effect on the net flow in Fig. 1 is then to "flatten" profile 2 into profile 3 making the velocity equal in high and low permeability regions. i.e. for viscosity $\mu$ and permeability $\kappa$ in high (subscript "hi") and low (subscript "lo") regions the ratio of which $(\kappa / \mu)$ is the proportionality coefficient governing velocity under the applied pressure gradient:

$$
u=\frac{\kappa_{\mathrm{hi}}}{\mu_{\mathrm{hi}}} \frac{\mathrm{d} p}{\mathrm{~d} x}=\frac{\kappa_{\mathrm{lo}}}{\mu_{\mathrm{lo}}} \frac{\mathrm{d} p}{\mathrm{~d} x}
$$

This flattening of the profile yields improved volume sweep of the reservoir. From the type of behaviour shown in Fig. 2, it is also important that the applied shear rates resulting from the pressure drop be rather low with a corresponding low Darcy velocity. This is not a problem since previous studies have demonstrated this effect at applied pressure gradients up to $0.25 \mathrm{bar} / \mathrm{cm}$ (Golombok et al. 2008). This is much higher than the actual pressure gradients during industrial oil recovery where typical pressure gradients are a factor of 10-100 less than this.

These shear-induced structure additives have only previously been studied in Couette cell or flow test units where they show shear thickening over only a restricted range of applied shear rate (Wang 1994; Cressely and Hartmann 1998). In these studies, at higher shear rates, the viscosity returns to the base fluid value. Similarly, our earlier study showed flow retardation only over a restricted range of high permeabilities with no retardation at the high

Fig. 2 Pressure drop as a function of velocity during core flooding. The linear profile shows a Darcy profile with water, and the positive deviation curve shows the response with additivated water. The net effect is a retardation of the flow at the reference pressure

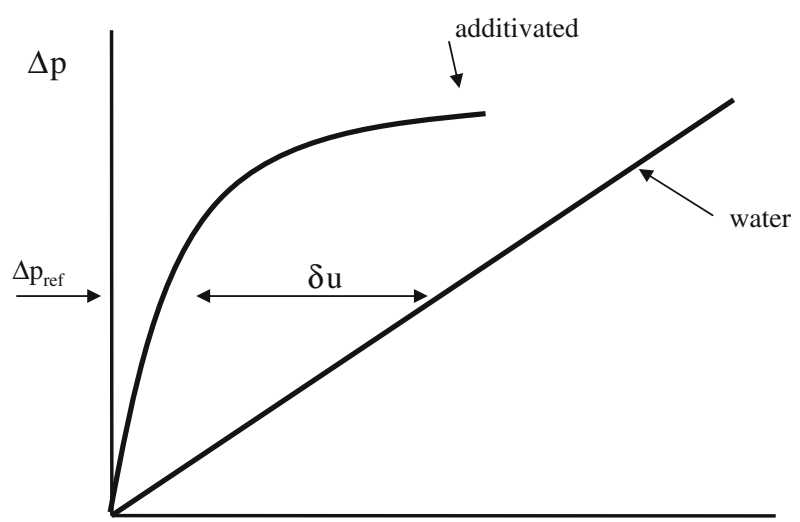


shear rates associated with (production) well bore flow. This manifests itself in Fig. 2 as a reduction in the flow at an identical applied pressure which goes back to its linear value when water is restored. The shear rate is classically defined in a geometry such as a Couette cell where a velocity gradient is imposed on a segment of fluid i.e. a velocity gradient is defined across a segment of fluid perpendicular to the direction of flow. Thus, although somewhat characterised for flow in tubes and cells, where shear rate is well defined, there has been no analysis for flow in porous media. (Berret et al. 1998). The application within permeable flow_particularly the extremely non-regular anisotropic oil reservoirs - is more problematic because of a lack of a mapping of permeability to the shear rate (and thus to the generated viscosity change which determines the flow). General relationships between shear rate and permeability remain a relatively obscure area of fluids engineering (Dullien 1979; Savins 1970; Greenkorn 1983). There is no clear way of associating a permeability and the shear rate in a rock pore generated by an applied pressure gradient.

In a simple hydraulic radius model, then we may identify the shear rate with the velocity gradient across the hydraulic radius $r_{\mathrm{H}}=\sqrt{ } \kappa$. This gives a shear rate term

$$
\gamma=\frac{u}{\sqrt{\kappa}}
$$

A more refined derivation takes into consideration the non-Newtonian parameters to yield (Teeuw and Hesselink 1980):

$$
\gamma=f(n) \frac{4 u}{\sqrt{8 \kappa \varphi}}
$$

where $f(n)$ is dependent on the form of the non-linearity of which Eq. 1 is one expression. The forms of Eqs. 3 and 4 are found in numerous studies in fluid flow in permeable mediahowever, they are only applicable in systems where the flow rate (and thus fluid velocity) is given. It has been previously noted (Dullien 1979) that much of the reported experimental work is carried out in terms of constant flow rate experiments because constant pressure drop runs appear unstable. Thus different paths through a permeable medium have identical fluid velocities in this representation.

In oil recovery operations, however, the fluid velocity is actually a dependent variable and it is the pressure drop which is applied. Other work (Slattery 1967; Canella et al. 1988) derives a similar form to Eq. 4 but explicitly relates shear rate and permeability in non-Newtonian flow

$$
\gamma=f(n) \sqrt{\frac{2 \kappa}{\phi}} \frac{1}{\mu} \frac{\partial p}{\partial x}
$$

Thus from the standpoint of applied pressure differences through a permeable medium, we expect a monotonically increasing relationship between shear rate and permeability. This applies to the range of permeabilities found in that reservoir and which we have previously demonstrated (Golombok et al. 2008). In the least permeable part of the reservoir, flow is slow and thus $\gamma$ is small. In the more permeable region, flow is retarded. Near the well bore (or in the extreme at the bore itself) the viscosity returns to the base fluid value. The viscosity as a function of shear rate (or equivalently permeability) follows a Lorentzian "resonance" type profile whose amplitude and resonance shear rate response maximum can be "tuned" by varying the concentration and the relative ratio of the surfactant and cosolute salt. An understanding of the shear and permeability relationship is the key to improving secondary recovery from oil reservoirs with novel rheological additives. The simple physical models discussed here provide a way to engineer the fluid front to overcome channelling and sweep 
more oil ahead of the water flood. We are currently developing these systems for a number of different reservoir and oilfield water flooding applications.

Open Access This article is distributed under the terms of the Creative Commons Attribution Noncommercial License which permits any noncommercial use, distribution, and reproduction in any medium, provided the original author(s) and source are credited.

\section{References}

Berret, J.-F., Gamez-Corrales, R., Oberdisse, J., Walker, L.: M., Lindner, P.: Flowstructure relationship of shearthickening surfactant solutions. Europhys. Lett. 41(6), 677 (1998). doi:10.1209/epl/i1998-00213-1

Canella, W.J., Huh, C., Seright, R.S.: Prediction of xanthan rheology in porous media. SPE 18089 63rd SPE Annual Fall Conference, p. 2. SPE, Houston (1988)

Cressely, R., Hartmann, V.: Rheological behaviour and shear thickening exhibited by aqueous CTAB micellar solutions. Eur. Phys. J. B 6, 57 (1998). doi:10.1007/s100510050526

Dullien, F.A.L.: Porous Media-Fluid Transport and Pore Structure. Academic Press, New York (1979)

Golombok, M., Crane, C., Ineke, E., Harris, J., Welling, M.: Novel additives to retard permeable flow. Exp. Therm. Fluid Sci. 32, 1499 (2008). doi:10.1016/j.expthermflusci.2008.04.001

Greenkorn, R.A.: Flow Phenomena in Porous Media. Marcel Dekker, New York (1983)

Jones, W.M.: Polymer additives in reservoir flooding for oil recovery: shear thinning or shear thickening? J. Phys. D Phys. 13(5), L87-L88 (1980)

Savins, J.G.: Non-Newtonian Flow Through Porous Media In: Flow Through Porous Media. ACS, Washington DC (1970)

Slattery, J.C.: Flow of viscoelastic fluids through porous media. A.I. Ch. E. J. 13(6), 1066-1071 (1967)

Teeuw, D., Hesselink, F.T.: Power Law Flow and Hydrodynamic Behaviour of Biopolymer Solutions in Porous Media. SPE 8982 5th Int. Symp. on Oilfield and Geothermal Chemistry, p. 28. SPE, Houston (1980)

Turcott, D., Schubert, G.: Geodynamics, Applications of Continuum Physics to Geological Problem. Cornell University Press, Ithaca (1982)

Wang, S.-Q., Hu, Y., Jamieson, A.M.: Formation of nonequilibrium micelles in shear and elongational flow, In: Structure and Flow in Surfactant Solutions, ACS Symposium Series 578, Washington (1994) 